\title{
A Generalized Radial Flow Model for Hydraulic Tests in Fractured Rock
}

\author{
J. A. BARKer \\ British Geological Survey, Wallingford, Oxfordshire, United Kingdom
}

\begin{abstract}
Models commonly used for the analysis of hydraulic test data are generalized by regarding the dimension of the flow to be a parameter which is not necessarily integral and which must be determined empirically. Mathematical solutions for this generalized radial flow model are derived for the standard test conditions: constant rate, constant head, and slug tests. Solutions for the less common, sinusoidal test are contained within the general solutions given. Well bore storage and skin are included and the extension to dual-porosity media outlined. The model is presented as a model of fractured media, for which it is most likely to find application because of the problem of choosing the appropriate flow dimension.
\end{abstract}

\section{INTRODUCTION}

Consider a typical hydraulic test in fractured rock, where water is injected between packers into an interval of a borehole known to contain at least one fracture. The problem that naturally arises when analyzing data from such a test is that of choosing an appropriate geometry for the fracture system into which flow occurs. If the fracture density is large and the distribution is isotropic, then a three-dimensional spherical flow geometry might be considered appropriate. If the fracture density is low or the system is very anisotropic, a one- or two-dimensional flow model would probably be preferred. However, it will often (perhaps normally) be the case that no presumption about the dimension of the flow system can be made with confidence.

This problem of choosing a dimension arose during the analysis of data from crosshole sinusoidal tests performed in the Stripa mine in Sweden. In that case attempts were made to use one, two- and three-dimensional models (Figure 1), both with and without dual porosity [Black et al., 1986]. None of the models was clearly superior, or gave a satisfactory representation of the whole data set. After considering a variety of possible variations on the models it was concluded that the most natural variation was to generalize the flow dimension to nonintegral values, while retaining the assumptions of radial flow and homogeneity. The resulting model is referred to as the generalized radial flow (GRF) model.

The primary aim of this paper is to provide a mathematical description of the GRF model leading to a comprehensive set of equations describing head changes during all of the commonly employed forms of hydraulic test. Conceptual problems associated with the model are discussed and related to the practical problems of applying it to field data.

These difficulties leave room to doubt the value of a model which represents flow in nonintegral dimensions; however, it is hoped that the sceptical reader will at least find interest in the generalization of familiar results in two dimensions (e.g., the Theis and Thiem equations) to one and three dimensions.

\section{Copyright 1988 by the American Geophysical Union.}

Paper number $88 \mathrm{WR} 03042$.

0043-1397/88/88WR-03042\$05.00

\section{GRF MODEL}

\subsection{Assumptions}

Listed below are the main assumptions made in developing the generalized radial flow model. Symbols are defined as they appear, but a full list of notation is also provided.

1. Flow is radial, $n$-dimensional flow from a single source into a homogeneous and isotropic fractured medium, characterized by a hydraulic conductivity $K_{f}$ and specific storage $S_{s f}$. (Generalization to the case of a dual-porosity medium is given in section 2.4.)

2. Darcy's law applies throughout the system.

3. The source is an $n$-dimensional sphere (projected through three-dimensional space; e.g., a finite cylinder in two dimensions, Figure $1 b$ ) of radius $r_{w}$ and storage capacity $S_{w}$ (the volumetric change in storage which accompanies a unit change in head).

4. The source has infinitesimal skin which is characterized by a skin factor $s_{f}$ : the head loss across the surface of the source is proportional to $s_{f}$ and the rate of flow through the surface.

5. Any piezometers in the fracture system have negligible size and storage capacity.

Throughout the mathematical development $r$ will be used to represent radial distance from the centre of the source measured in the fracture flow system. The real (Euclidian) distance from the source must therefore equal $r$ divided by the tortuosity, which can be regarded as an empirical parameter.

\subsection{Flow Equations}

Consider the region bounded by two equipotential surfaces which have radii $r$ and $r+\Delta r$. These surfaces are the projections of $n$-dimensional spheres through three dimensional space by an amount $b^{3-n}$. For example, when $n$ is equal to two the surfaces are finite cylinders of length $b$ (Figure $1 b$ ). A sphere of radius $r$ has an area $\alpha_{n} r^{n-1}$, where $\alpha_{n}$ is the area of a unit sphere in $n$ dimensions:

$$
\alpha_{n}=2 \pi^{n / 2} / \Gamma(n / 2)
$$

and $\Gamma(x)$ is the gamma function. (Some specific values of $\alpha_{n}$ are given in Table 1.) The region between the equipotential shells must therefore have a volume $b^{3-n} \alpha_{n} r^{n-1} \Delta r$, where $\Delta r$ is small. 
(a)

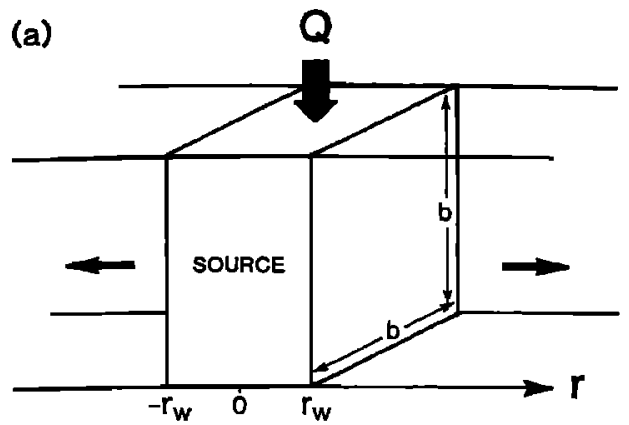

(b)

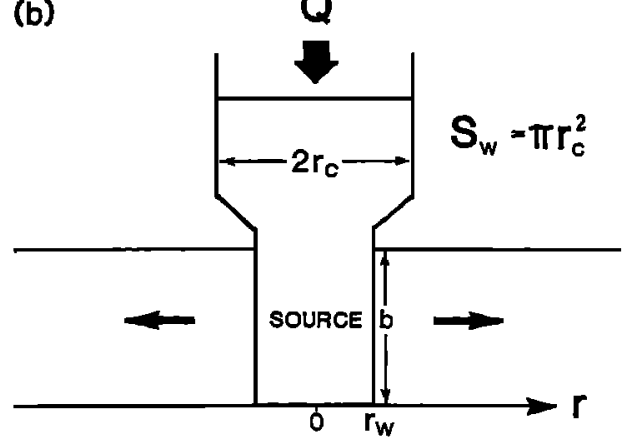

(c)

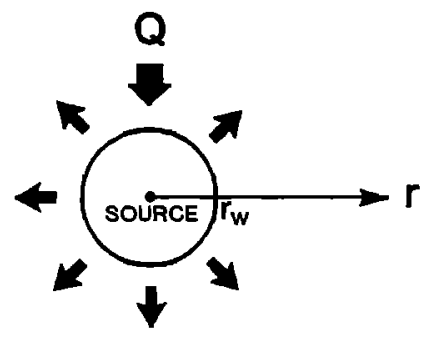

Fig. 1. Flow geometries for integral dimensions: $(a)$ onedimensional flow from a plane $\left(n=1, v=\frac{1}{2}\right)$; (b) two-dimensional flow from a cylinder (borehole) $(n=2, v=0)$; and (c) threedimensional flow from a sphere $\left(n=3, v=-\frac{1}{2}\right)$.

Suppose that during a small period $\Delta t$ the head in this shell changes by $\Delta h$, so the volume of water entering the shell must be

$$
\Delta V=S_{s f} b^{3-n} \alpha_{n} r^{n-1} \Delta r \Delta h
$$

which follows from the definition of specific storage.

From Darcy's law, the net volumetric flow rate into the shell is

$$
q=K_{f} b^{3-n} \alpha_{n}\left[(r+\Delta r)^{n-1} \partial h / \partial r(r+\Delta r, t)-r^{n-1} \partial h / \partial r(r, t)\right]
$$

where $K_{f}$ is the hydraulic conductivity of the fracture system and $h(r, t)$ is the head.

The conservation equation for water in the shell takes the simple form

$$
\Delta V=q \Delta t
$$

which, on substituting from (2) and (3) and taking limits, becomes

$$
S_{s f} \frac{\partial h}{\partial t}=\frac{K_{f}}{r^{n-1}} \frac{\partial}{\partial r}\left(r^{n-1} \frac{\partial h}{\partial r}\right)
$$

It will be assumed that water is injected into the source at a volumetric rate $Q(t)$. Water will also flow between the source and the fracture system at a rate given by Darcy's law. So the rate of change of storage in the source is described by

$$
S_{w} \hat{c} H / \hat{c} t(t)=Q(t)+K_{f} b^{3-n} x_{n} r_{w^{*}}{ }^{n-1} \hat{c} h / \hat{c} r\left(r_{w}, t\right)
$$

where $H(t)$ is the head in the source and $S_{w}$ is the storage capacity of the source.

The head within the source is assumed to differ from that in the formation at radius $r_{w}$ due to a skin of infinitesimal thickness which impedes the flow:

$$
H(t)=h\left(r_{w}, t\right)-s_{f} r_{w} \hat{c} h / \hat{c} r\left(r_{w}, t\right)
$$

where $s_{f}$ is the skin factor. The form of (7) has been chosen such that $s_{f}$ is dimensionless and has the standard interpretation for two-dimensional, cylindrical flow where it is normally encountered [e.g., Ramey, 1982].

A boundary condition is introduced which states that the head is constant at a fixed distance from the source

$$
h\left(r_{0}, t\right)=h_{0}
$$

In the majority of cases considered this condition takes the special form of zero head at infinite distance from the source.

It will normally be assumed that the initial condition is that the head is zero throughout the system:

$$
h(r, 0)=H(0)=0
$$

This condition does not exclude the slug test (section 5), but will not apply to the steady state sinusoidal test (section 6).

\subsection{General Solution of the Flow Equations}

A solution to the above equations is now derived in the form of relationships between the quantities $h(r, t), H(t)$, and $Q(t)$. For brevity, the solution is restricted to the case of zero head at infinity for a finite source but is otherwise general. One case of a fixed head at a finite distance for an infinitesimal source is considered in section 3.2. The general solution is presented in the form of Laplace transforms from which timedependent results can be obtained by numerical inversion (see Appendix B).

The Laplace transform of (5) is, using the initial condition (9).

$$
p S_{s f} \bar{h}(r, p)=\frac{K_{f}}{r^{n-1}} \frac{d}{d r}\left(r^{n-1} \frac{d \bar{h}}{d r}\right)
$$

TABLE 1. Values for Integral and Half Integral Dimensions

\begin{tabular}{lrll}
\hline$n$ & $\nu$ & $\alpha_{n}$ & $\phi_{r}(z)$ \\
\hline 0 & 1 & 0 & $\frac{z K_{0}(z)}{K_{1}(z)}$ \\
$\frac{1}{2}$ & $\frac{3}{4}$ & $0.734 \ldots$ & \\
1 & $\frac{1}{2}$ & 2 & $z$ \\
$\frac{3}{2}$ & $\frac{1}{4}$ & $3.85 \ldots$ & \\
2 & 0 & $2 \pi$ & $\frac{z K_{1}(z)}{K_{0}(z)}$ \\
$\frac{5}{2}$ & $-\frac{1}{4}$ & $9.22 \ldots$ & $\frac{(1+z)}{z}$ \\
3 & $-\frac{1}{2}$ & $4 \pi$ & \\
\hline
\end{tabular}


where $p$ is the transform variable (see equation (A27) in Appendix A, which presents a collection of useful formulae).

The general solution of $(10)$ is

$$
\bar{h}(r, p)=C(p) r^{v} K_{v}(\lambda r)+D(p) r^{v} I_{v}(\lambda r)
$$

where $C(p)$ and $D(p)$ are functions to be determined from the boundary conditions

$$
\begin{aligned}
v & =1-n / 2 \\
\lambda^{2} & =p S_{s f} / K_{f}
\end{aligned}
$$

and $K_{v}(z)$ and $I_{v}(z)$ are modified Bessel functions. (Take particular note of (12), since many of the results given in this paper are expressed in terms of $v$ rather than the dimension $n$.)

Introducing the restricted boundary condition

$$
\lim _{r \rightarrow \infty} h(r, t)=0
$$

gives

$$
D(p)=0
$$

so

$$
\bar{h}(r, p)=C(p) r^{\nu} K_{v}(\lambda r)
$$

The derivative of the head at the surface of the source is obtained using (A13):

$$
d \bar{h} / d r\left(r_{w,} p\right)=-C(p) \lambda r_{w}{ }^{v} K_{v-1}\left(\lambda r_{w}\right)
$$

Taking the Laplace transform of (7), and using (15) and (16) gives

$$
\bar{H}(p)=C(p) r_{w}{ }^{v} K_{v}\left(\lambda r_{w}\right)+s_{f} C(p) \lambda r_{w}{ }^{1+\nu} K_{v-1}\left(\lambda r_{w}\right)
$$

from which the function $C(p)$ can be determined as

$$
C(p)=r_{w}{ }^{-v} \bar{H}(p) /\left[K_{v}(\mu)+s_{f} \mu K_{v-1}(\mu)\right]
$$

where

$$
\mu=\lambda r_{w}
$$

Taking the Laplace transform of (6) and using (9), (16), and (19) gives

$$
p S_{w} \bar{H}(p)=\bar{Q}(p)-K_{f} b^{3-n} \alpha_{n} C(p) r_{w}{ }^{-v} \mu K_{v-1}(\mu)
$$

Then substituting for $C(p)$ from (18) gives a relationship between the source head and the injection rate:

$$
\bar{Q}(p) / \bar{H}(p)=p S_{w}+K_{f} b^{3-n^{3}} \alpha_{n} r_{w}{ }^{n-2} \Phi_{v}(\mu) /\left[1+s_{f} \Phi_{v}(\mu)\right]
$$

where the function $\Phi_{v}(z)$ is defined by

$$
\Phi_{v}(z)=z K_{v-1}(z) / K_{v}(z)
$$

Combining (15) and (17) gives the relationship between the source head and the head in the fracture system:

$$
\bar{h}(r, p) / \bar{H}(p)=\frac{\rho^{v} K_{v}(\mu \rho) / K_{v}(\mu)}{1+s_{f} \Phi_{v}(\mu)}
$$

where

$$
\rho=r / r_{w}
$$

Finally, eliminating $\tilde{H}(p)$ from (21) and (23) gives the relationship between the injection rate and the head in the fracture system:

$$
\begin{aligned}
\bar{h}(r, p) / \bar{Q}(p)=\rho^{v} K_{v}(\mu \rho) / K_{v}(\mu) \\
\cdot\left[p S_{w}\left[1+s_{f} \Phi_{v}(\mu)\right]+K_{f} b^{3-n} \alpha_{n} r_{w}{ }^{n-2} \Phi_{v}(\mu)\right]^{-1}
\end{aligned}
$$

In summary, (21), (23), and (25) provide the required Laplace transform solution to the flow equations in the form of relationships between $h, H$, and $Q$. Which equation is of particular interest will depend on the form of test under consideration, as will be seen from the special cases considered later.

\subsection{Extension to Dual-Porosity Media}

The above derivations have been based on the assumption that the fracture system can be regarded as a homogeneous medium, characterized by a hydraulic conductivity $K_{f}$ and specific storage $S_{s f}$. The solutions can be readily extended to include dual-porosity media by replacing (13) by

$$
\lambda^{2}=p S_{s f}[1+\sigma B(\xi)] / K_{f}
$$

where $\sigma$ is the ratio of matrix storage to fracture storage per unit volume,

$$
\xi^{2}=p S_{s m} a^{2} / K_{m}
$$

where $S_{s m}$ and $K_{m}$ are the specific storage and hydraulic conductivity of the matrix material, and $a$ is the volume to area ratio of the matrix blocks.

The function $B(\xi)$ characterizes the shape of the matrix blocks and has been termed the block geometry function (BGF) [Barker, 1985b, c]. Most BGF's that have been employed belong to the family of functions:

$$
B_{\theta}(\xi)=\xi^{-1} I_{\theta / 2}(\theta \xi) / I_{\theta / 2-1}(\theta \xi)
$$

which corresponds to planar, cylindrical, and spherical block shapes when $\theta$ is equal to 1,2 , and 3 , respectively. Further extension to include fracture skin [e.g., Moench, 1984] is possible, and the interested reader should consult Barker [1985c] for details.

\section{Constant Rate Tests}

The first special case considered is the constant rate test, which will include generalizations of the formulae normally attributed to Theis, Thiem, and Jacob. Using the Laplace transform given by (A28) gives

$$
\bar{Q}(p)=Q_{0} / p
$$

if water is injected at a constant rate $Q_{0}$ beginning at time zero. Note the convention that $Q_{0}$ is positive for injection of water which gives positive heads, since the initial condition is that of zero head. If water is being extracted then the following results remain unchanged, but $h$ must be interpreted as drawdown.

\subsection{Infinitesimal Source and Infinite Flow Region}

Using (29) in (25), and setting well storage to zero gives

$$
\bar{h}(r, p)=\frac{Q_{0} r^{\nu} K_{v}(\lambda r)}{p K_{f} b^{3-n} \alpha_{n} K_{v-1}(\mu) \mu^{1-\nu} \lambda^{\nu}}
$$

Now letting the well radius (and hence $\mu$ ) tend to zero and using (A8) and (A9) gives

$$
\bar{h}(r, p)=\frac{Q_{0} r^{v} K_{v}(\lambda r)}{p K_{f} b^{3-n} \alpha_{n} \lambda^{v} 2^{-v} \Gamma(1-v)} \quad v<1
$$

This Laplace transform can be inverted analytically using (A32), and then using (A16) and (A34)

$$
h(r, t)=\frac{Q_{0} r^{2 v}}{4 \pi^{1-\nu} K_{f} b^{3-n}} \Gamma(-v, u) \quad v<1
$$




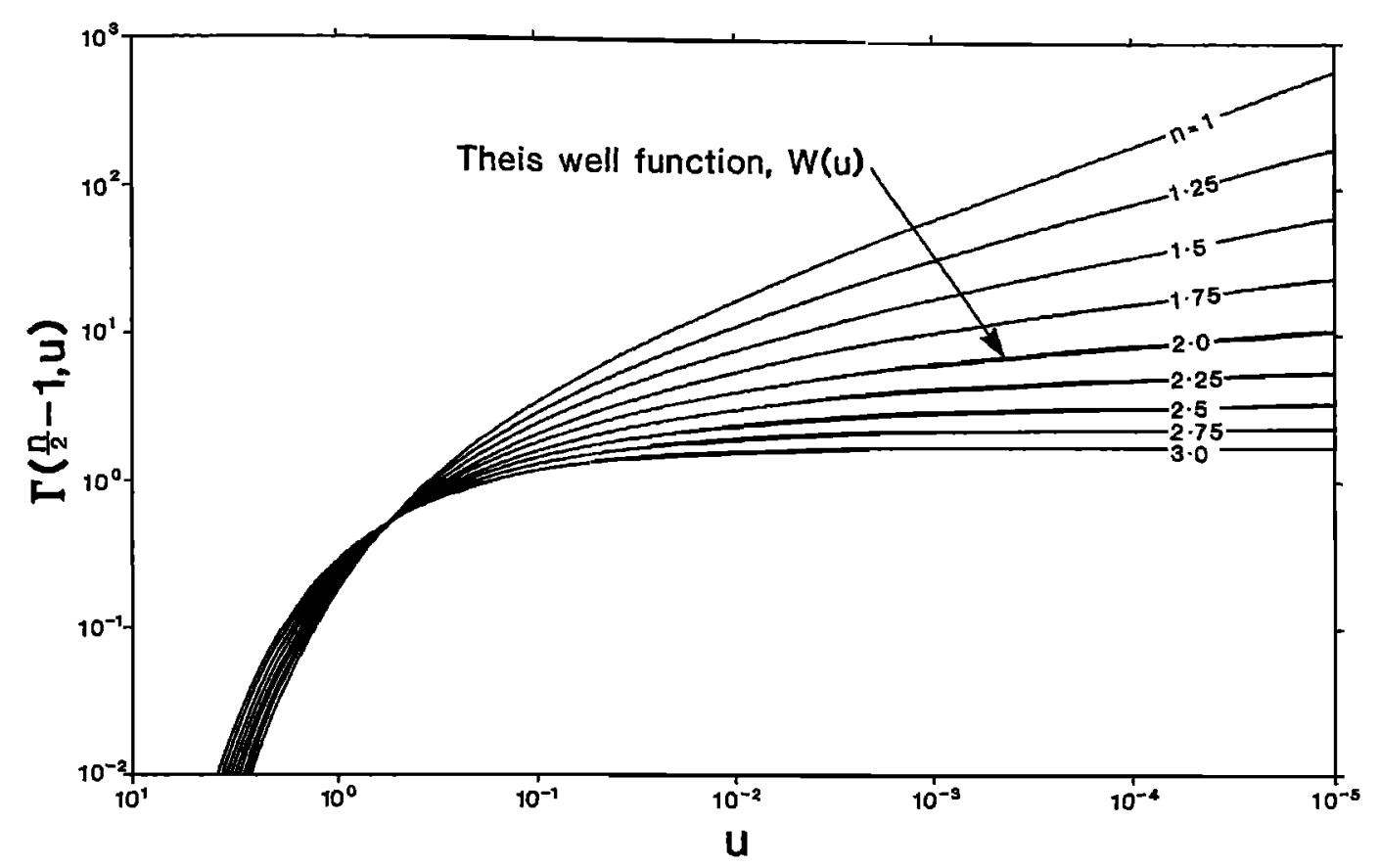

Fig. 2. Incomplete gamma function (generalized Theis well function).

where

$$
u=S_{s f} r^{2} / 4 K_{f} t
$$

and $\Gamma(a, x)$ is the (complementary) incomplete gamma function which is shown in Figure 2.

Equation (32) is a generalization of the equation given by Theis [1935] for radial flow with a line source. An even more general form of (32) was derived by Shchelkachev [1971] for the case of a pumping rate which is proportional to $t$ raised to an integral power (but only for integral dimensions). Using the special cases of the gamma function given in (A24)-(A26) gives

$$
\begin{aligned}
& h(r, t)=\frac{Q_{0} r}{2 \sqrt{\pi} K_{f} b^{2}}\left(\frac{e^{-u}}{\sqrt{u}}-\sqrt{\pi} \text { erfc } \sqrt{u}\right) \quad n=1 \\
& h(r, t)=\frac{Q_{0}}{4 \pi K_{f} b} E_{1}(u) \quad n=2 \\
& h(r, t)=\frac{Q_{0}}{4 \pi K_{f} r} \operatorname{erfc} \sqrt{u} \quad n=3
\end{aligned}
$$

in which erfe is the complimentary error function and $E_{1}$ is the exponential integral. Equation (34b) is the Theis equation, which is almost invariably written using the notation $W(u)$ instead of the exponential integral. Equation (34a) was derived by Miller [1962].

In Figure 2, which is a log-log plot, it appears that many of the curves tend to straight lines as $u$ tends to zero (time tends to infinity). This behavior can be investigated by considering the asymptotic form of (32) which, using (A22), gives

$$
\begin{array}{r}
h(r, t)=\frac{Q_{0}}{4 \pi^{1-v} K_{f} b^{3-\pi v}}\left[\left(\frac{4 K_{f} t}{S_{s f}}\right)^{v}-\Gamma(1-v) r^{2 v}\right] \\
v \neq 0 \quad(n \neq 2)
\end{array}
$$

which can be regarded as a generalization of the Jacob equation [Cooper and Jacob, 1946].
Note that in (35) the time-dependent term dominates for large times when $n$ is less than two; this explains the linear behavior in Figure 2 and also shows that the slopes of the lines tend to $v(=1-n / 2)$. Also, note that when $v<0(n>2)$ the time-dependent term tends to zero; hence the steady state will be achieved when and only when the dimension is greater than two.

The integral dimension cases of (35) are

$$
\begin{array}{ll}
h(r, t)=\frac{Q_{0} r}{2 K_{f} b^{2}}\left(\left(\frac{K_{f} t}{\pi S_{s f} r^{2}}\right)^{1 / 2}-1\right) & n=1 \\
h(r, t)=\frac{Q_{0}}{4 \pi K_{f} b}\left[\ln \left(\frac{4 K_{f} t}{S_{s f} r^{2}}\right)-;\right] & n=2 \\
h(r, t)=\frac{Q_{0}}{4 \pi K_{f} r}\left(1-r\left(\frac{S_{s f}}{\pi K_{f} t}\right)^{1 / 2}\right) & n=3
\end{array}
$$

where $\gamma$ is Euler's constant. Equation (36b) is the Jacob equation and is obtained from (35) using (A18) and

$$
\lim _{\varepsilon \rightarrow 0}\left(a^{\varepsilon}-b^{c}\right) / \varepsilon=\ln a / b
$$

\subsection{Infinitesimal Source With a Fixed Head Boundary}

The case now considered is that of an infinitesimal source with a fixed head $h_{0}$, boundary condition at a finite radius $r_{0}$. This case is of interest because, for the two-dimensional case, it must leads to the Thiem equation in the steady state, and also because it can be used for comparison with various types of numerical model, where it is easier to simulate a finite rather than infinite system.

Using the limiting form of (6), the boundary condition at the source:

$$
Q_{0} / p=-\lim _{r_{w} \rightarrow 0} K_{f} b^{3-n} x_{n} r_{w}{ }^{n-1} \partial \hat{h} / \partial r\left(r_{w}, p\right)
$$

along with the fixed head boundary condition given in $8,(11)$ 
becomes

$$
\begin{aligned}
\bar{h}(r, p)=h_{0} / p-Q_{0} r^{*}\left[2^{1-v} \pi^{-v} b^{3-n} K_{f} p \lambda^{v}\right]^{-1} \\
\cdot\left(\frac{K_{v}\left(\lambda r_{0}\right) I_{v}(\lambda r)-I_{v}\left(\lambda r_{0}\right) K_{v}(\lambda r)}{\pi I_{v}\left(\lambda r_{0}\right)+2 \sin (v \pi) K_{v}\left(\lambda r_{0}\right)}\right)
\end{aligned}
$$

where use has been made of (A4), (A5), (A8), (A9), (A13), (A17) and (A34).

The steady state head distribution in this case can be obtained either from the asymptotic behavior of (39) or, more readily, by putting zero specific storage in (5) and applying the fixed head boundary condition. The result is

$$
h(r)-h_{0}=\frac{Q_{0} \Gamma(1-v)}{4 \pi^{1-v} K_{f} b^{3-n_{v}}}\left(r_{0}{ }^{2 v}-r^{2 v}\right) \quad v \neq 0
$$

which is the generalization of the Thiem equation. It can also be regarded as a special case of the generalization of Darcy's law, described by Narasimhan [1985]. Equation (40) might have been inferred directly from (35). The specific cases of interest are

$$
\begin{aligned}
& h(r)-h_{0}=\frac{Q_{0}}{2 K_{f} b^{2}}\left(r_{0}-r\right) \quad n=1 \\
& h(r)-h_{0}=\frac{Q_{0}}{2 \pi K_{f} b} \ln \frac{r_{0}}{r} \quad n=2 \\
& h(r)-h_{0}=\frac{Q_{0}}{4 \pi K_{f}}\left(\frac{1}{r}-\frac{1}{r_{0}}\right) \quad n=3
\end{aligned}
$$

Equation (41b), obtained by applying (37), is the Thiem equation [Thiem, 1906]; it is sometimes referred to as the Dupuit formula.

\subsection{Finite Source in an Infinite Domain}

Considering the more general case of section 3.1 where the source has a finite radius, it is only necessary to substitute the Laplace transform of the injection rate (29), into (21) and (25) to obtain the solution. Extension to dual-porosity media (section 2.4) gives generalizations of the solutions presented by Moench [1984] and Barker [1985a], which were themselves generalizations of previous results.

As time tends to infinity, the head in the fractures tends to that given by the generalized Theis equation (and subsequent$1 y$, the Jacob equation) given in section 3.1. From (7) and (35) the head in the source is therefore related to the asymptotic fracture head by

$$
H(t)=\left(1-2 v s_{f}\right) h\left(r_{w}, t\right) \quad v<0
$$

for dimensions greater than two, when the system tends to a steady state. In terms of the injection rate, the steady state head in the source $H(\infty)$ is given by

$$
H(\infty)=-\frac{Q_{0} \Gamma(1-v)\left(1-2 v s_{f}\right)}{4 \pi^{1-v} K_{f} b^{3-n} v} \quad n>2
$$

The only special case of interest is that for spherical flow:

$$
H(\infty)=\frac{\left(1+s_{f}\right) Q_{0}}{4 \pi r_{w} K_{f}} \quad n=3
$$

which could readily be derived from Darcy's law and (7).

\section{Constant Head Tests}

Suppose that the head in the source is held at a constant value $H_{0}$ for all times greater than zero. Using (A28), the
Laplace transform of $H(t)$ is given by

$$
\bar{H}(p)=H_{0} / p
$$

which can be used in (21) and (25) to give the injection rate (required to maintain this head) and the fracture system head, respectively. After long times the injection rate will tend to a constant value for dimensions greater than two, then the source head to injection rate ratio is given by (43). For dimensions less than two the injection rate tends asymptotically to zero, while the head in the fractures (at any finite radius) tends to that of the source.

\section{Slug Tests}

Slug tests (sometimes referred to as a pulse tests) are initiated by a sudden change of head $H_{i}$, in the source zone: a variety of method are employed to bring about this change. Since the storage capacity of the source is $S_{w}$ for unit change in head, these tests can always be regarded as starting with an injection (or abstraction) of a volume $S_{w} H_{i}$ of water. Assuming this process to be effectively instantaneous, the effective injection rate is therefore

$$
Q(t)=S_{w} H_{i} \delta(t)
$$

The Dirac delta function $\delta(t)$ has a Laplace transform of unity, and this can be used in (21) to give the head in the source as

$\bar{H}(p)=S_{w} H_{i} /\left\{p S_{w}+K_{f} b^{3-n^{n}} \alpha_{n} r_{w}{ }^{n-2} \Phi_{v}(\mu) /\left[1+s_{f} \Phi_{v}(\mu)\right]\right\}$

which when inverted numerically will give the slug test response curves. (The equation for the head in the fractures is readily obtained, but is normally of little interest.)

For large values of $\mu$ (which correspond to small values of the dimensionless time $K_{f} t / S_{s f} r_{w}{ }^{2}$ ) and zero skin factor, (47) becomes

$$
\bar{H}(p)=H_{i} /(p+\beta \sqrt{p})
$$

where

$$
\beta=b^{3-n} \alpha_{n} r_{w}{ }^{n-1}\left(S_{s f} K_{f}\right)^{1 / 2} / S_{w}
$$

Equation (48) can be inverted analytically, using (A31), to give

$$
H(t)=H_{i} e^{\beta 2 t} \operatorname{erfc}(\beta \sqrt{t})
$$

which generalizes a previously known result for the twodimensional limiting case [Bredehoeft and Papadopulos, 1980]. Incidentally, $(50)$ can be used to show that the dimensionless slope, $d\left(H / H_{i}\right) / d \ln t$, of a slug test curve, at any given recovery level, is independent of the dimension in this asymptotic region. Therefore it should be expected that the analysis of slug test data would often fail to produce a unique dimension.

\section{Sinusoidal Tests}

A sinusoidal test [Black and Kipp, 1981] can be performed using either a controlled injection rate or a controlled head in the source. The Laplace transforms of these boundary conditions are immediately given by (A30), and they can then be substituted into (21), (23), or (25) to give the required Laplace transform solution (which can then be inverted numerically to derive the transient response).

In practice it is normally found that the observed fracture heads tend rapidly toward steady state conditions (constant amplitude and phase shift). Therefore for the purposes of data analysis, only steady state solutions are required. Such solutions could be obtained by considering the asymptotic behav- 


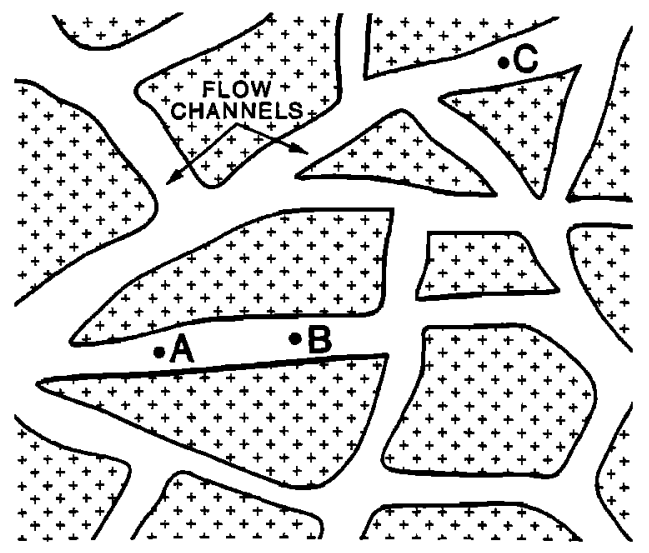

Fig. 3. Hypothetical fractured medium with channels.

ior of the transient solutions just described; however, a simpler approach to obtaining the same results will be outlined.

Assuming purely periodic behavior throughout the system:

$$
\begin{aligned}
h(r, t) & =h_{\omega}(r)^{i \omega t} \\
H(t) & =H_{\omega} e^{i \omega t} \\
Q(t) & =Q_{\omega} e^{i \omega t}
\end{aligned}
$$

where $h_{\omega}, H_{\omega}$, and $Q_{\omega}$ are independent of $t$; in general, they are complex which allows the modulus of each to represent amplitude while the argument represents phase shift.

When (51) to (53) are substituted into the basic flow equations (5), (6), and (7), and the exponential terms cancelled, the resulting equations are identical to the Laplace transforms of (5) 7 ) provided:

$$
\begin{aligned}
p & \equiv i \omega \\
\bar{h}(r, p) & \equiv h_{\omega}(r) \\
\bar{H}(p) & \equiv H_{\omega} \\
\bar{Q}(p) & \equiv Q_{\omega}
\end{aligned}
$$

Therefore (21), (23), and (25) can be used directly to give steady state sinusoidal solutions. For example, for a controlled sinusoidal injection rate, $Q_{\omega}$ can be set equal to the amplitude of the injection rate (no phase shift), and (54), (55), and (57) can be used in (25) to give an equation for $h_{\omega}$, the response of the fracture system. If this result is simplified by setting the skin factor to zero and the dimension to two, the result given by Black and Kipp [1981] is obtained.

\section{Discussion}

\subsection{Application to Fractured Rock}

There are two reasons for proposing the GRF model as a candidate for use in simulating hydraulic tests in fractured rock. First, it contains few parameters yet permits a great range of behavior patterns; this makes it particularly suitable for data analysis. Second, since the dimension of the flow system is difficult to choose, it is a natural choice for generalization. Even if it were to be demonstrated that the flow dimension must assume integral values, the GRF model is still of value in that it provides a single formulation for all three dimensions. There is no intention to suggest that this model should replace existing models, some of which have special characteristics [e.g., Hsieh, 1983; Karasaki, 1986] which makes them a more obvious choice under certain circumstances.

\subsection{Parameters}

The GRF model introduces two novel parameters: the dimension $n$ and the extent of the flow zone $b$. Tortuosity is also introduced, but is used in the familiar sense to represent the ratio of the length of the flow path between two points and the distance between the same points.

The parameter $b$ is difficult to describe for nonintegral flow dimensions, but it has a simple interpretation for integral values. For one-dimensional flow it is simply the square root of the flow area (Figure 1a). For two-dimensional flow $b$ is the extent of the flow region perpendicular to the plane of flow (for example, the thickness of a confined aquifer, Figure $1 b$ ). For spherical flow the parameter has no significance: this is possible mathematically because $b$ appears raised to the power of $3-n$ in the flow equations, so this term reduces to unity when $n=3$.

While it is natural to characterize the hydrogeological properties of rock by its hydraulic conductivity and specific storage, the general solution given by (21), (23), and (25) suggests the use of the diffusivity $K_{f} / S_{s f}$ and the quantity $b^{3-n} K_{f}$ (which becomes the transmissivity in the two-dimensional case). Indeed, to extract any two of the parameters contained in these groups (not counting the dimension $n$ which appears independently in the equations) the third must be known a priori.

The meaning of the dimension $n$ in the case of nonintegral values is probably the most difficult conceptual feature of the model. This value may only be meaningful within the context of more fundamental models. For example, it might be expected that the dimension would be related to a characteristic statistical property of a fracture network model. (Work on investigating such a relationship has already begun). A reasonable conjecture would be that radial diffusion (Brownian motion) on a fractal network of dimension $n$ would be described by (5). And these two ideas are related through the observation that real fracture networks appear to have fractal properties [e.g., Long et al., 1985].

The dimension does not appear to be an intrinsic hydraulic property of the fracture system, which is obviously an unsatisfactory feature of the model. Consider the hypothetical system depicted in Figure 3, where water flows in an infinite system of channels within a plane. Imagine a test where water is injected at point $\mathrm{A}$, the pressure response at point $\mathrm{B}$ might reasonably be expected to be (approximately) characteristic of flow in a linear system, at least for early times. However, for a longterm test, the pressure response at point $C$ (and possibly also at point B) would be more likely to be characteristic of cylindrical flow. This scale dependence of the dimension is somewhat analogous to that of the dispersion coefficient used in transport modeling, which, nevertheless, has proved to be a useful parameter.

The dimension is not related to any angular restriction on the flow direction. For example, steady state flow within a cone from a point source at its apex would be truly spherical flow $(n=3)$.

The dimension is not related to the space-filling characteristics of the flow paths. For example, a spiral flow channel could fill a plane but would have the flow characteristics of a linear system. In general, it seems necessary (but perhaps not 
sufficient) that for flow systems to have different dimensions they must have different topologies.

\subsection{Anisotropy}

The GRF model does not appear to permit the introduction of anisotropy in cases of nonintegral dimension (since a conductivity tensor must have an integral number of terms). Because of the tendency of rocks to fracture along particular directions, a homogeneous model with anisotropy [e.g., Hsieh, 1983] will often be more valid than the GRF model, particularly when the fracture density is high.

\subsection{Finite Source}

Several solutions have been given which relate to an ideal n-dimensional spherical source (notably the slug test solution). In practice, such a source cannot be realized except for integral dimensions. So the head in a real source cannot be expected to correspond closely to the value of $H$, although it could be hoped that variations in $H$ might be indicative of real head changes. Because of this difficulty the model is more applicable to interference tests than to single borehole tests.

\subsection{Sinusoidal Tests}

Although the GRF model was originally developed for the analysis of data from sinusoidal tests, there are special difficulties in applying it to such tests. A single test results in only two numbers (a phase shift and an amplitude) so several tests are required to obtain a complete set of model parameters. Further information can be obtained by varying the frequency of the test [Black et al., 1986]; however, the derived dimension is likely to decrease with increasing frequency, since the radius of influence of the test will decrease. Tests over various distances can also be used, but again the problem of dimension varying with scale arises.

\subsection{Relationship to Other Models}

The main reason for carrying out hydraulic tests is usually to obtain parameter values that can be used in some form of regional flow model. If the GRF model reveals an integral dimension for a test then the only problem is that of deciding the orientation of the flow system when the dimension is either one or two. However, for nonintegral dimensions the model, along with any parameters derived using it, is not consistent with any commonly used model. It is hoped, however, that it will be possible to establish a relationship between the GRF model and fracture network models.

\section{Conclusions}

Mathematical solutions have been derived for the commonly used forms of hydraulic test for an arbitrary flow dimension. These solutions include generalizations of many well-known formulae used for pumping test analysis, including the Theis equation, (32), the Jacob equation, (35), and the Thiem equation (40).

The more general solutions are expressed in terms of the Laplace transforms of the time-dependent heads and injection rate. The solutions include well bore skin and, in section 2.4 , it is shown that these are easily extended to include dual porosity. Normally, it will be necessary to invert these transforms numerically; a brief discussion of methods is given in Appendix B.

Constant rate tests in an infinite medium will tend to a steady state for all dimensions greater than two. Steady state solutions are diagnostic of the dimension via the radial variation in head. When the dimension is less than or equal to two, the test will be transient; when it is less than two, the dimension is obtained directly from the slope of a plot of the logarithm of head against the logarithm of time, for large times.

The model is a straightforward extension of integral dimension models and it therefore appears to be a natural candidate for a model of flow in fractured rock, where the appropriate dimension is often uncertain. However, significant theoretical and practical difficulties arise in the application of the model; notably, the dimension does not appear to be an intrinsic property of the rock (and is likely to be scale-dependent), the idealized spherical source geometry cannot be realized, and anisotropy cannot be included. Also, the physical interpretation of the flow dimension is unclear, although it is conjectured that the model respresents radial diffusion on a fractal network. Numerical experiments using fracture network models should help to give some insight into these problems.

\section{ApPendix A: Mathematical FormulaE}

This appendix provides a set of useful mathematical formulae which have been drawn from various sources, often with some modification. Most are employed within the derivations in this paper, but others will be of value either in simplifying the formulae in special cases or in evaluating the results.

Unless otherwise stated: the symbols $z$ and $\lambda$ represent complex numbers, $v, a$, and $n$ are real (possibly integer) numbers, and $N$ is an integer.

Modified Bessel Function of the First Kind: $I_{v}(z)$

$$
\begin{aligned}
& I_{v}(z)=e^{-(1 / 2) v \pi i} J_{v}\left(z e^{(1 / 2) \pi i}\right) \quad-\pi<\arg z \leq \frac{1}{2} \pi \\
& =e^{(3 / 2) v \pi i} J_{v}\left(z e^{-(3 / 2) \pi i}\right) \quad \frac{1}{2} \pi<\arg z \leq \pi \\
& I_{v-1}(z)-I_{v+1}(z)=2 v I_{v}(z) / z \\
& I_{-N}(z)=I_{N}(z) \\
& \lim z^{-v} I_{v}(z)=2^{-v} / \Gamma(1+v) \quad v \neq-1,-2, \cdots \\
& =\rightarrow 0 \\
& \frac{d}{d z} z^{v} I_{v}(\lambda z)=\lambda z^{v} I_{\nu-1}(\lambda z)
\end{aligned}
$$

Macdonald (Bessel) Function: $K_{v}(z)$

$$
\begin{gathered}
K_{v}(z)=\frac{\pi}{2}\left[I_{-v}(z)-I_{v}(z)\right] / \sin (v \pi) \quad v \neq \text { integer } \\
K_{N}(z)=\lim _{v \rightarrow N} K_{v}(z) \\
K_{-v}(z)=K_{v}(z) \\
\lim _{z \rightarrow 0} z^{v} K_{v}(z)=2^{v-1} \Gamma(v) \quad v>0 \\
\lim _{z \rightarrow 0}\left[z^{-v} K_{v}(z)-2^{v-1} \Gamma(v) z^{-2 v}\right]=-2^{-v-1} \Gamma(1-v) / v
\end{gathered}
$$

$$
\lim _{v \rightarrow 0} \frac{\partial}{\partial v} K_{v}(z)=0
$$

$\lim _{z \rightarrow \infty}\left(\frac{2 z}{\pi}\right)^{1 / 2} e^{z} K_{v}(z)=1$ 


$$
\begin{gathered}
d / d z z^{v} K_{v}(\lambda z)=-\lambda z^{v} K_{v-1}(\lambda z) \\
K_{1 / 2}(z)=\left(\frac{\pi}{2 z}\right)^{1 / 2} e^{-z} \\
K_{3 / 2}(z)=\left(\frac{\pi}{2 z}\right)^{1 / 2}(1+1 / z) e^{-z}
\end{gathered}
$$

Gamma Function: $\Gamma(v)$

$$
\begin{gathered}
\Gamma(1+v)=v \Gamma(v) \\
\Gamma(v) \Gamma(1-v)=\pi \csc (\pi v) \\
\lim _{x \rightarrow 0} \frac{\ln \Gamma(1-x)}{x}=\gamma \\
\Gamma\left(\frac{1}{2}\right)=\sqrt{\pi} \\
\Gamma(1)=1 \\
\Gamma\left(\frac{3}{2}\right)=\frac{1}{2} \sqrt{\pi}
\end{gathered}
$$

Incomplete Gamma Function: $\Gamma(a, x)$

$$
\Gamma(a, x)=\Gamma(a)-\sum_{m=0}^{\infty} \frac{(-1)^{m} x^{a+m}}{m !(a+m)} \quad a \neq 0,-1,-2, \cdots
$$

$$
\begin{gathered}
\partial \Gamma(a, x) / \partial x=-x^{a-1} e^{-x} \\
\Gamma(0, x)=E_{1}(x) \\
\Gamma\left(\frac{1}{2}, x\right)=\sqrt{\pi} \operatorname{erfc} \sqrt{x} \\
\Gamma\left(-\frac{1}{2}, x\right)=2\left(e^{-x} / \sqrt{x}-\sqrt{\pi} \operatorname{erfc} \sqrt{x}\right)
\end{gathered}
$$

Laplace Transforms

$$
\begin{gathered}
L\{f(t)\}=\bar{f}(p)=\int_{0}^{\infty} e^{-p t} f(t) d t \\
L\{1\}=1 / p \\
L\{\delta(t)\}=1 \\
L\{\sin \omega t\}=\omega /\left(\omega^{2}+p^{2}\right) \\
L\left\{e^{a 2 t} \operatorname{erfc}(a \sqrt{t})\right\}=1 /[\sqrt{p(a+\sqrt{p})]} \\
L\left\{\frac{1}{2}\left(\frac{a}{2}\right)^{v} \Gamma\left(-v, \frac{a^{2}}{4 t}\right)\right\}=p^{-(v / 2)-1} K_{v}(a \sqrt{p}) \\
\lim _{p \rightarrow 0} p L\{f(t)\}=\lim _{t \rightarrow \infty} f(t)
\end{gathered}
$$

Unit Sphere in $n$ Dimensions

$$
\begin{aligned}
& \text { area }=\alpha_{n}=2 \pi^{n / 2} / \Gamma(n / 2) \\
& \text { volume }=\pi^{n / 2} / \Gamma(1+n / 2)
\end{aligned}
$$

See aiso Table 1.

\section{APPENDix B: Evaluation of FormulaE}

Many of the results given in this paper are expressed in the form of Laplace transforms of the time-dependent functions of interest. To evaluate such a result at a specific time it is necessary to invert the transform. Analytical inversion is generally impractical so numerical inversion must be employed. Even if analytical inversion were possible, the resulting formulae would normally be so complex that they would be more difficult and computationally expensive to evaluate directly than by numerical inversion of their transforms.

The most commonly used Laplace transform inversion al- gorithm is that given by Stehfest [1970]; however, the author has found the method described by Talbot [1979] to be superior both in terms of speed and accuracy.

To employ the Talbot algorithm the transform function must be expressed in terms of a complex transform variable $p$, and this requires the evaluation of Bessel functions of complex argument. These complex functions are also required to evaluate the sinusoidal solutions described in section 6 even though no transform inversion is involved. The CERN Subroutine Library [Strassen, 1975] provides a routine for the evaluation of $J_{v}(z)$, and using (A1), (A6), and (A7) this can provide routines adequate for evaluating all of the formulae given in this paper. However, a recently developed package of routines [Amos, 1986] provides a more accurate and efficient set of functions and is strongly recommended.

\section{NOTATION}

a volume to area ratio for a matrix block.

$b$ extent of the flow region (e.g., Figure 1).

$B(\xi)$ block geometry function for dual-porosity media.

erfc $(x)$ complementary error function.

$E_{1}(u)$ exponential integral (Theis well function).

$h(r)$ steady state head in fracture system.

$h(r, t)$ transient head in fracture system.

$h_{0}$ fixed head in fractures at radius $r_{0}$.

$h_{\omega}(r)$ (complex) head in fractures due to a sinusoidal source.

$H(t)$ head in source.

$H_{i}$ initial head in source during a slug test.

$H_{0}$ source head during a constant head test.

$H_{\text {to }}$ (complex) source head during a sinusoidal test.

$i \sqrt{-1}$.

$I_{v}(z)$ modified Bessel function.

$J_{v}(z)$ Bessel function.

$K_{f}$ hydraulic conductivity of the fracture system.

$K_{m}$ hydraulic conductivity of the rock matrix.

$K_{v}(z)$ modified Bessel function.

$n$ dimension of the fracture flow system.

p Laplace transform variable.

$q$ volumetric flow rate of water.

$Q(t)$ volumetric rate of injection into the source.

$Q_{0}$ volumetric rate of injection during a constant rate test.

$Q_{\omega} \quad$ (complex) amplitude of the injection rate during a sinusoidal test.

$r$ radial distance from the centre of the source (measured along the flow paths).

$r_{0}$ radius at which a fixed head $h_{0}$ is specified.

$r_{w}$ radius of the source.

$s_{f}$ skin factor, defined by (7).

$S_{s f}$ specific storage of the fracture system.

$S_{\mathrm{sm}}$ specific storage of the rock matrix.

$S_{w}$ storage capacity of the source.

$t$ time.

$u=S_{s f} r^{2} / 4 K_{f} t$.

$V$ a volume of water.

$W(u)$ Theis well function.

$\alpha_{n}$ area of a unit sphere in $n$ dimensions.

$\beta$ see (49).

$\gamma \quad$ Euler's constant $(=0.5772 \ldots)$.

$\Gamma(x)$ gamma function.

$\Gamma(a, x) \quad$ (complementary) incomplete gamma function. 


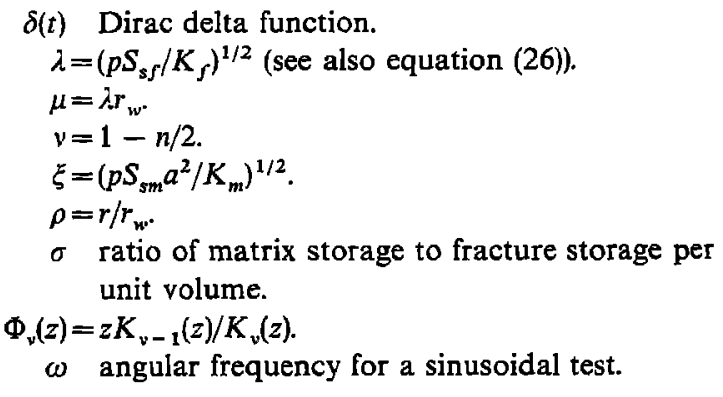

Acknowledgments. This work was motivated by the hydraulic testing programme carried out in the Stripa mine, and I would like to acknowledge the work of my colleagues on that programme; discussions with John Black and David Noy were particularly stimulating. Brief discussions with Jane Long and Kenzi Karasaki (Lawrence Berkeley Laboratory) were a valuable source of encouragement. The comments of the referees were also appreciated. This paper is published with the approval of the Director, British Geological Survey (NERC).

\section{REFERENCES}

Amos, D. E., Algorithm 664: A portable package for Bessel functions of a complex argument and nonnegative order, ACM Trans. Math. Software, I2(3), 265-273, 1986.

Barker, J. A., Generalized well-function evaluation for homogeneous and fissured aquifers, $J$. Hydrol., 76, 143-154, $1985 a$.

Barker, J. A., Block-geometry functions characterizing transport in densely fissured media, J. Hydrol., 77, 263-279, 19856 .

Barker, J. A., Modelling the effects of matrix diffusion on transport in densely fissured media, Mem. Int. Assoc. Hydrogeol., 18, 250-269, $1985 c$.

Black, J. H., J. A. Barker, and D. J. Noy, Crosshole investigations: the method, theory and analysis of crosshole sinusoidal pressure tests in fissured rock, Stripa Proj., Int. Rep. 86-03, SKB, Stockholm, 1986.

Black, J. H., and K. L. Kipp, Determination of hydrogeological parameters using sinusoidal tests: A theoretical appraisal, Water Resour. Res., 17(3), 686-692, 1981.

Bredehoeft, J. D., and S. S. Papadopulos, A method for determining the hydraulic properties of tight formations, Water Resour. Res, 16(1), 233-238, 1980.

Cooper, H. H., Jr., and C. E. Jacob, A generalized method for evaluating formation constants and summarizing well-field history, Eos Trans. $A G U, 27,526-534,1946$.

Hsieh, P. A., Theoretical and field studies of fluid flow in fractured rocks, Ph.D. dissertation, 200 pp., Univ. of Ariz., Tucson, 1983.

Karasaki, K., Well test analysis in fractured media, Ph.D. dissertation, 239 pp., Univ. of Calif., Berkeley, 1986.

Long, J. C. S., H. K. Endo, K. Karasaki, L. Pyrak, P. MacLean, and P. A. Witherspoon, Hydrologic behavior of fracture networks, Mem. Int. Assoc. Hydrogeol., 17, 449-462, 1985.

Miller, F. G., Theory of unsteady-state inflow of water in linear reservoirs, J. Inst. Pet., 48, 467-477, 1962.

Moench, A. F., Double-porosity models for a fissured groundwater reservoir with fracture skin, Water Resour. Res., 20(7), 831-846, 1984.

Narasimhan, T. N., Geometry-imbedded Darcy's law and transient subsurface flow, Water Resour. Res., 21(8), 1285-1292, 1985.

Ramey, H. J., Jr., Well-loss function and the skin effect: A review, in Recent Trends in Hydrogeology, edited by T. N. Narasimhan, Geological Society of America, Boulder, Colo., 1982.

Shchelkachev, V. N., A general solution for the differential equations of one-dimensional nonstationary flows in a multidimensional space, New's Acad. Sci. USSR: Liqud Gas Mech., 3, 481-487, 1971.

Stehfest, H., Numerical inversion of Laplace transforms, Commun $A C M, 13(1), 47-49,1970$.

Strassen, H. H., Double precision complex Bessel function $J_{v}(z)$, Routine C332, CERN Computer Centre Program Library, Geneva, 1975.

Talbot, A., The accurate numerical inversion of Laplace transforms, $J$. Inst. Math. Appl., 23, 97-120, 1979.

Theis, C. V., The relation between the lowering of the piezometric surface and the rate and duration of discharge of a well using ground water storage, Eos Trans. AGU, 16, 519-524, 1935.

Thiem, G., Hydrologische Methoden, J. M. Gebhardt, Leipzig, 1906.

J. A. Barker, British Geological Survey, Maclean Building, Crowmarsh Gifford, Wallingford, Oxfordshire, OX1O 8BB United Kingdom.

(Received February 17, 1988; revised May 25, 1988: accepted June $3,1988$. ) 